\title{
The association of EGF rs2237051 variant, serum EGF levels and generalized aggressive periodontitis: a preliminary study
}

\author{
Xian'e Wang ${ }^{1}$, Wenjing Li ${ }^{1}, \mathbf{L i ~ X u}{ }^{1}$, Ruifang Lu ${ }^{1}$, Huanxin Meng ${ }^{\text {Corresp. } 1}$ \\ 1 Department of Periodontology, National Engineering Laboratory for Digital and Material Technology of Stomatology, Beijing Key Laboratory of Digital \\ Stomatology, Peking University School and Hospital of Stomatology, Beijing, China \\ Corresponding Author: Huanxin Meng \\ Email address: kqhxmeng@bjmu.edu.cn
}

Background: Epidermal growth factor (EGF) is a pro-inflammatory small peptide that stimulates cell growth, proliferation and differentiation through binding to its receptor. EGF rs2237051 and serum EGF levels have been demonstrated to be related with a variety of diseases, including several tumors and inflammatory diseases. Therefore, this study aims to investigate the association of the EGF rs2237051 variant and serum EGF levels in Chinese patients with generalized aggressive periodontitis (GAgP).

Material and Methods: A case-control study was conducted among 216 patients with GAgP and 138 healthy controls. The clinical parameters of plaque index (PLI), probing depth (PD), attachment loss (AL) and bleeding index (BI) were recorded. The EGF rs2237051 polymorphism was genotyped using time-offlight mass spectrometry, and serum EGF levels were determined. Logistic and linear regression models were used to investigate the association between the genotypes of EGF rs2237051, serum EGF levels, and GAgP risk.

Results: The AA genotype of EGF rs2237051 showed higher risk for GAgP than the combined genotypes GG and $A G$ (adjusted $O R=1.65,95 \% \mathrm{Cl}: 1.06,2.57$ ). Increased serum EGF levels were associated with GAgP (adjusted OR $=1.18,95 \% \mathrm{Cl}: 1.14,1.22$ ). Moreover, the serum EGF level for the AA genotype was significantly higher than that for the AG/GG genotypes in patients with $\mathrm{GAgP}$ (adjusted $\beta=4.70,95 \% \mathrm{Cl}$ : $2.09,7.31)$.

Conclusion: We demonstrated that EGF rs2237051 variant and the increased level of serum EGF were associated with the risk of GAgP, the serum EGF was up-regulated in patients with GAgP. It was indicated that serum EGF might be a biomarker of GAgP, and EGF rs2237051 may be related to the genetic background of GAgP. 
1 The association of EGF rs2237051 variant, serum EGF levels and generalized aggressive

2 periodontitis: a preliminary study

3 Xian'e Wang ${ }^{1}$, Wenjing $\mathrm{Li}^{1}$, Li Xu${ }^{1}$, Ruifang $\mathrm{Lu}^{1}$, Huanxin Meng ${ }^{1}$

$4{ }^{1}$ Department of Periodontology, National Engineering Laboratory for Digital and Material

5 Technology of Stomatology, Beijing Key Laboratory of Digital Stomatology, Peking University

6 School and Hospital of Stomatology, Beijing, China

7 Corresponding author: Huanxin Meng.

8 Department of Periodontology, Peking University School and Hospital of Stomatology. 22

9 Zhongguancun Nandajie, Haidian District, 100081, Beijing, China. Fax: 86-10-62173402

10 E-mail: kqhxmeng@bjmu.edu.cn 


\section{Abstract}

13 Background: Epidermal growth factor (EGF) is a pro-inflammatory small peptide that stimulates cell growth, proliferation and differentiation through binding to its receptor. EGF rs2237051 and serum EGF levels have been demonstrated to be related with a variety of diseases, including several tumors and inflammatory diseases. Therefore, this study aims to investigate the association of the EGF rs2237051 variant and serum EGF levels in Chinese patients with generalized aggressive periodontitis (GAgP).

Material and Methods: A case-control study was conducted among 216 patients with GAgP and 138 healthy controls. The clinical parameters of plaque index (PLI), probing depth (PD), attachment loss (AL) and bleeding index (BI) were recorded. The EGF rs2237051 polymorphism was genotyped using time-of-flight mass spectrometry, and serum EGF levels were determined. Logistic and linear regression models were used to investigate the association between the genotypes of EGF rs2237051, serum EGF levels, and GAgP risk.

Results: The AA genotype of EGF rs2237051 showed higher risk for GAgP than the combined genotypes $\mathrm{GG}$ and $\mathrm{AG}$ (adjusted $\mathrm{OR}=1.65,95 \% \mathrm{CI}: 1.06,2.57$ ). Increased serum EGF levels were associated with GAgP (adjusted OR $=1.18,95 \% \mathrm{CI}: 1.14,1.22$ ). Moreover, the serum EGF level for the AA genotype was significantly higher than that for the AG/GG genotypes in patients with GAgP (adjusted $\beta=4.70,95 \%$ CI: 2.09, 7.31).

Conclusion: We demonstrated that $E G F$ rs2237051 variant and the increased level of serum EGF were associated with the risk of GAgP, the serum EGF was up-regulated in patients with GAgP. It was indicated that serum EGF might be a biomarker of GAgP, and EGF rs2237051 may be related to the genetic background of GAgP. 


\section{Introduction}

Generalized aggressive periodontitis (GAgP) is an infectious disease characterized by severe and rapid alveolar bone destruction, which can eventually lead to tooth loss in otherwise healthy, relatively young adults. Although the direct cause for GAgP is bacterial infection, the progression and severity of the disease depend upon the interaction between host genes and environmental factors (Ozer Yucel et al., 2015; Vieira \& Albandar, 2014). Lots of studies have demonstrated that genetic factors play an important role in the pathogenesis of aggressive periodontitis (Vieira \& Albandar, 2014).

Epidermal growth factor (EGF), a polypeptide molecule, is a multifactorial cytokine that exerts its biological effects, including cell growth, cell proliferation, and wound repair, through binding to cell surface receptors. EGF can stimulate the secretion of collagenase, gelatinase, and plasminogen activators in mucosal keratinocytes. Moreover, EGF can regulate extracellular matrix degradation (Lyons et al., 1993). Severe periodontitis has been shown to be associated with increased activity of collagenase and gelatinase (Birkedal-Hansen, 1993). Chang et al. (1996) demonstrated that EGF-binding capacity in human gingival tissue was enhanced by nearly three-fold during inflammation compared with that in non-inflamed gingiva. Hormia et al. (1993) have demonstrated that the mean EGF concentration in stimulated saliva is slightly higher in juvenile patients with periodontitis than that in healthy controls and proposed that this elevation may be associated with the pathogenesis mechanism of juvenile periodontitis. In addition, it has been reported that the level of serum EGF in patients with rapid progressive periodontitis is significantly higher than that in the healthy controls (Pietruska, Pietruski \& Stokowska, 2000). Hence, EGF may be an important mediator in the pathogenesis of periodontitis.

Given the importance of EGF in periodontal diseases, the investigation of genetic polymorphisms that may affect its transcriptional activity can provide important information on its function in periodontal diseases. $E G F$ rs2237051 polymorphism is a non-synonymous singlenucleotide polymorphism (SNP) in the coding region of the $E G F$ gene that causes a change from isoleucine (ATA) to methionine (ATG) at amino acid position 708. The rs2237051 polymorphism has been demonstrated to be related with several types of cancers, such as lung cancer (Hosgood et al., 2008), advanced esophageal squamous cell carcinoma (Yang et al., 2014) and gastric cancer (Zhan et al., 2013). The rs2237051 polymorphism not only promotes the risk of cancer, but also affects their clinical outcomes. EGF and genetic factors are important for the regulation of the pathogenesis of periodontitis and GAgP, respectively. It is meaningful to 
67 investigate the interrelationship between the functional EGF rs2237051 polymorphism and $68 \mathrm{GAgP}$, which maybe further enrich the genetic background of GAgP.

69 Serum EGF levels have been found to be associated with a variety of diseases, including 70 Parkinson's disease, several tumors and inflammatory diseases. Considering that periodontal 71 inflammation may cause systemic immune inflammatory response (Cardoso et al.,2018), it is 72 necessary to study whether EGF changes in the serum of patients with aggressive periodontitis and the effect of $E G F$ rs2237051 polymorphism on the serum EGF concentration.

Therefore, we aimed this study to investigate the association between the $E G F$ rs 2237051 variant with GAgP, assess the correlation between serum EGF levels and GAgP, and determine the effect of the rs2237051 genotypes on the serum EGF concentration in patients with GAgP.

\section{Material and methods}

\section{Subject population}

In the present case-control study, 216 Chinese patients with GAgP and 138 periodontally healthy controls were enrolled. Patients were from the Department of Periodontology at the Peking University School and Hospital of Stomatology and the controls were volunteers from the staff and student population of the hospital. The following clinical and radiographic criteria proposed by the 1999 International World Workshop for a Classification of Periodontal Diseases and Conditions were applied for the diagnosis of GAgP (Armitage, 1999):

- $\quad$ systematically healthy, except for periodontal disease;

- $\leq 35$ years of age when diagnosed;

- a minimum of 8 teeth with probing depth (PD) $>5 \mathrm{~mm}$ and attachment loss (AL) $>3 \mathrm{~mm}$, and a minimum of 3 teeth should not be first molars or incisors among them.

Inclusion criteria for the controls were: $\leq 35$ years of age; $\mathrm{PD} \leq 3 \mathrm{~mm}$ and on obvious clinical attachment loss; $\leq 10 \%$ of sites with a bleeding index $(\mathrm{BI}) \geq 2$.

Exclusion criteria for all subjects were: $\geq 36$ years old; smoker; history of periodontal treatment or antimicrobial therapy within 6 months; being pregnant for females; systemic diseases.

This study was approved by ethic committee of Peking University Health Science Center (NO.0313) and was conducted in accordance with the Helsinki Declaration of 1975, as revised in 2013. All subjects had informed written consent questionnaire for the study. BMI (body mass index) is calculated by weight $/$ height $^{2}$. 


\section{Assessment of clinical parameters}

The plaque index (PLI) was scored for buccal and lingual surfaces of all teeth, except the third molars, according to the Quigley \& Hein (1962) plaque index. PD and AL were measured six sits (mesial, middle and distal sites of the buccal and lingual sites) per tooth except the third molar using Williams periodontal probe. The greatest BI values of the buccal and lingual surfaces were recorded 30 seconds after probing (Mazza, Newman \& Sims, 1981). All the clinical periodontal parameters were recorded by two skilled periodontal specialists (Dong Shi \& Li Xu). The calibration was performed on 10 patients with GAgP. The consistency of the replicated measurements of PLI, PD, AL and BI for each examiner (intra-calibration) and paired measurements between the pair of two periodontal specialists (inter-examiner calibration) were recorded. Of the replicated measurements for each examiner, 94.0\% (Dong Shi) and 96\% (Li Xu) were within 1 unit for PLI; 97.0\% (Dong Shi) and 95.8\% (Li Xu) were within $1 \mathrm{~mm}$ for PD; and 91.5\% (Dong Shi) and 93.2\% (Li Xu) were within $1 \mathrm{~mm}$ for AL, and 97.0\% (Dong Shi) and 98.0\% (Li Xu) were within 1 unit for BI. Of the paired measurements between the two examiners (Dong Shi versus $\mathrm{Li} \mathrm{Xu}$ ), 92.0\% were within 1unit for PLI; 89.8\% were within $1 \mathrm{~mm}$ for AL, 93.5\% were within $1 \mathrm{~mm}$ for PD; and 91.6\% were within 1 unit for BI.

\section{Blood collection and assessment of serum EGF levels}

A fasted peripheral blood sample was collected and distributed into two tubes from all subjects using venipuncture tubes between 8:00 a.m. and 10:00 a.m. The tube with EDTA (ethylenediaminetetraacetic acid, EDTA) was used for white blood cells separation and genomic DNA isolation and the other one without EDTA was applied for measuring serum EGF levels. After 1 hour of clotting at $20-26^{\circ} \mathrm{C}$, serum and white blood cells were separated from the blood by centrifugation. The white blood cells and serum were immediately stored at $-70{ }^{\circ} \mathrm{C}$ until use.

Serum EGF levels were assessed using an enzyme-linked immunosorbent assay kit (R\&D Systems, Minneapolis, MN), according to the manufacturer's instructions. The experiment was performed in duplicate.

\section{DNA extraction and genotyping}

The blood DNA mini kit (Watson Biotechnologies Inc., Shanghai, China) was used for the extraction of genomic DNA from white blood cells, according to the manufacturer's instructions. The EGF rs2237051 single nucleotide polymorphism was genotyped through SEQUENOM MassARRAY matrix-assisted laser desorption ionization time-of-flight (MALDI-TOF) mass spectrometry (Sequenom, San Diego, CA, USA). The genotyping was performed by one investigator who was blind to the periodontal status of all participants. 
131 The primers for the EGF rs2237051 genotyping were designed through the Assay Designer

132 software package (Sequenom):

133 Forward: 5'-ACGTTGGATGGAGGATTATGTGTGGTTCTC-3'

134 Reverse: 5'-ACGTTGGATGGTACTCTATCTTTGCCAGTC-3'

135 All genotyping was performed blind to clinical diagnosis by a single investigator.

\section{Statistical analysis}

137 Continuous variables are presented as mean $\pm \mathrm{SD}$, and categorical variables are shown as $\mathrm{N}(\%)$.

138 The differences between patients with GAgP and healthy controls were assessed using one-way 139 ANOVA (normal distribution) or Mann-Whitney U (skewed distribution) test. Agreement of 140 genotype frequencies with Hardy-Weinberg equilibrium expectations was tested by using a $\chi^{2}$ 141 goodness-of-fit test for the controls. Univariate logistic analysis was used to determine the 142 association between the variables, such as age, gender, BMI, genotypes of rs2237051and serum $143 \mathrm{EGF}$, and risk for GAgP. Multiple logistic regression models were used to predict the adjusted 144 odds ratio (OR) at 95\% confidence intervals (CI) for the association of the EGF rs2237051 145 variant or serum EGF levels with the risk for GAgP with or without adjusting the covariables. 146 Linear regression models were used to estimate the adjusted coefficient ( $\beta$ ) at 95\% CI for the 147 association between $E G F$ rs2237051 polymorphism and serum EGF levels in healthy controls 148 and patients with GAgP. A two-tailed $\mathrm{P}<0.05$ was considered statistically significant. Statistical 149 analyses were performed using R (http:// www.R-project.org) and EmpowerStats software 150 (www.empow erstats.com, X\&Y Solutions Inc., Boston, MA, USA).

\section{Results}

The basic characteristics, clinical periodontal parameters, EGF rs2237051 genotypes, and serum EGF levels in patients with GAgP and healthy controls are shown in Table 1. The BMI (both continuous and categories) and periodontal parameters (mean PLI, PD, BI, and AL) were significantly higher in patients with GAgP than that in healthy controls $(\mathrm{P}<0.05)$. The distribution of the $E G F$ rs2237051 genotypes was significantly different between the GAgP and control groups. No evidence of deviation from Hardy-Weinberg equilibrium was found for the SNP of rs2237051 (the P value of goodness-of-fit test was $>0.05$ ). The level of serum EGF was significantly higher in the GAgP group than that in the control group $(46.77 \pm 9.38 \mathrm{pg} / \mathrm{ml}$ vs. $33.54 \pm 8.62 \mathrm{pg} / \mathrm{ml}$, respectively, $\mathrm{P}<0.001$ ). No significant differences were found in age, gender, and PLI between both groups.

The univariate analysis data for the risk of GAgP is shown in Table 2. When compared to the 
163 individuals with $\mathrm{BMI}<18.5 \mathrm{~kg} / \mathrm{m}^{2}$, individuals with $\mathrm{BMI} \geq 24.9 \mathrm{~kg} / \mathrm{m}^{2}$ has higher odds of 164 periodontitis $(\mathrm{OR}=3.29,95 \% \mathrm{CI}$ : $1.37,7.91)$, but no significant difference were found for the 165 individuals with $\mathrm{BMI} \geq 18.5$ and $<24.9 \mathrm{~kg} / \mathrm{m}(\mathrm{P}>0.05)$.

166 The risk for GAgP was significantly different for the different genotypes of EGF rs2237051. The 167 AG and AA genotypes of EGF rs2237051 increased the risk for GAgP by more than 2-fold and 3-fold ( $\mathrm{OR}=2.29$; 95\% CI: 1.04, 5.04; $\mathrm{OR}=3.26$, 95\% CI: 1.47, 7.22; respectively) compared to the GG genotype. The AA genotype showed a higher OR (62\%) than did the combined genotypes of $\mathrm{GG}$ and $\mathrm{AG}(\mathrm{OR}=1.62 ; 95 \% \mathrm{CI}$ : 1.05, 2.51). Moreover, serum EGF levels in patients with GAgP were higher than that in healthy controls. Consequently, the risk for GAgP was increased by $17 \%$ with one unit increase of serum EGF (OR=1.17; 95\% CI: 1.13, 1.21).

The multiple regression analysis data for the genotypes of $E G F$ rs2237051 and serum EGF levels for the risk of GAgP are presented in Table 3. The AA genotype showed a higher risk for GAgP than the combined genotypes of GG and AG (adjusted OR=1.65; 95\% CI: 1.06, 2.57) after adjusting for age, gender, and BMI. Moreover, increased level of serum EGF was found in the patients with GAgP after adjusting for covariables. The risk for GAgP was increased by $18 \%$ per unit with an increase in serum EGF levels (adjusted OR=1.18; 95\% CI: 1.14, 1.22).

The association between the genotypes of EGF rs2237051 and serum EGF level is shown in Table 4. Among all the participants, individuals with AA genotype of EGF rs2237051 had significant higher serum EGF concentration than the subjects with AG/GG genotype (adjusted $\beta=2.86$; 95\% CI: 0.88, 4.84) after adjustment of age, gender and BMI. While there was no significant difference in the serum EGF concentration among healthy controls with different genotypes of $E G F$ rs2237051, among the GAgP patients, individuals with AA genotype of $E G F$ rs2237051 had significant higher serum EGF level than the person with AG/GG genotype (adjusted $\beta=4.70 ; 95 \%$ CI: 2.09, 7.31).

187

188

189

190

No significant differences were found for the association between $E G F$ rs2237051 variants and periodontal parameters, including PLI, BI, PD and AL; and no significant differences were observed for the association between serum EGF levels and periodontal parameters (data not shown).

\section{Discussion}

In the present study, we have demonstrated that there is a significant association between the genotypes of EGF rs2237051 and risk for GAgP as well as serum EGF levels, and that serum EGF concentration is increased in patients with GAgP. Our results indicate that the variants of 
EGF rs2237051 are associated with GAgP in Chinese patients. To our knowledge, this is the first study to demonstrate that $E G F$ rs 2237051 is a susceptibility gene for GAgP. A previous study has reported the association between another intron-specific locus of EGF and severe chronic periodontitis (Suzuki et al., 2004).

The EGF rs2237051 (A/G) is a non-synonymous SNP in the coding region of the EGF gene which results in a change from isoleucine (ATA) to methionine (ATG) at amino acid position 708 (I708M). So far, the function of rs2237051 polymorphism has not been well understood and its role in different diseases is not consistent. Zhan et al. (2013) reported that the $\mathrm{G}$ allele carriers of rs2237051 is associated with an increased risk of gastric cancer. In another study, the variant carriers of $E G F$ rs2237051 was reported to be associated with a markedly decreased risk of lung cancer (Hosgood et al., 2008). In advanced esophageal squamous cell carcinoma patients, the variant allele $\mathrm{G}$ of $E G F$ rs2237051 was associated with favorable prognosis (Yang et al., 2014). In the present study, we found that the AA genotype of rs2237051 showed the higher serum EGF level and a higher OR (1.62) than did the combined genotypes of GG and AG in Chinese patients with GAgP. The effect of the rs2237051 polymorphism in our study was consisted with other studies (Hosgood et al.,2008; Yang et al.,2014). Amino acid alteration may influence the spatial conformation of the EGF protein and therefore alter the function of the gene. This may explain the correlation between the rs2237051 of the EGF gene and GAgP. The possible way via which the polymorphism could actually influence GAgP needs further investigation.

Our study found that serum EGF concentration is increased in patients with GAgP compared with controls. Actually, some studies have reported the association between EGF and periodontitis; however, the results are not consistent in gingival tissues, saliva, gingival crevicular fluid, and serum. Chang et al. (1996) demonstrated that EGF-binding capacity in human gingival tissue was enhanced by nearly three-fold during inflammation compared with that in non-inflamed gingiva while the EGF level in gingival crevicular fluid (GCF) was significantly lower in the samples collected from pockets $>5 \mathrm{~mm}$ than in those from pockets $<5 \mathrm{~mm}$. Elevated crevicular fluid flow, as a consequence of inflammation, may have a diluting effect on the EGF concentration in GCF. Hormia et al. (1993) have demonstrated that the mean EGF concentration in stimulated saliva is slightly higher in juvenile patients with periodontitis than that in healthy controls. Moosavijazi et al. (2014) demonstrated that the levels of salivary EGF in patients with advanced periodontitis is lower than that in the healthy subjects. However, the sample size of this study was relatively small and only 11 patients with advanced periodontitis, 13 patients with gingivitis and 16 healthy controls were consisted. The technique used to evaluate the results was also different with other studies (Hormia et al.,1993). Another study has shown that the level of serum EGF in patients with rapid progressive periodontitis was 
230 significantly higher than that in the healthy controls, which was consistent with our study 231 (Pietruska, Pietruski \& Stokowska, 2000). We speculated that the role of serum EGF in patients 232 with GAgP was pro-inflammatory. The possible ways via which EGF could influence 233 periodontitis included that EGF can stimulate IL-1 $\beta$ secretion to induce the production of IL-8 in 234 gingival fibroblasts, which in turn plays an important role in the pathogenesis of periodontitis by

235

236

237

238

239

240 increasing the recruitment of immune cells to the inflamed tissue (Yucel-Lindberg \& Brunius, 2006). In addition, another study showed that EGF can regulate the expression of MMP-1, $-3,-7$ and -11 in a dose-dependent manner. This suggests that EGF might play a role in periodontal destruction and wound repair (Cury et al., 2007). In this study, we showed that the level of serum EGF is increased in patients with GAgP, which confirms a positive correlation between serum EGF level and GAgP. The possible reason for the increase of serum levels of EGF in patients with GAgP could be either spillover from the gingival tissues to the peripheral circulation or it could be caused by a systemic inflammatory response to progressive disease in the periodontal pocket.

In our study, we found that when compared to the individuals with $\mathrm{BMI}<18.5 \mathrm{~kg} / \mathrm{m}^{2}$, individuals with $\mathrm{BMI} \geq 24.9 \mathrm{~kg} / \mathrm{m}^{2}$ has higher odds of aggressive periodontitis, which was consist with other studies (Moura-Grec et al.,2014; Ekuni et al.,2008). BMI was also reported to be associated with EGF (Caroleo et al., 2019), so it was regarded as a covariant to analyze the association among the rs2237051 variant, serum EGF and GAgP. However, no significant difference was found after adjusting for BMI. It suggested that the relationship among the rs2237051 variant, serum EGF and GAgP might not be influenced by BMI.

There are some limitations of this study. Firstly, the sample size of this study is limited due to the low incidence of aggressive periodontitis. In addition, according to the study design of epidemiology, the information bias and selection bias might exist in the present study. Besides, when interpreting the results, it must be considered that in order to match the age to GAgP patients, age of the healthy subjects was less than 36 years in the present study. These samples may potentially have been subjects with periodontitis but have not manifested signs and symptoms yet. Indeed, the periodontal healthy subjects should be observed for quite a long time to make sure that they are actually healthy. Furthermore, only one functional SNP of EGF was included in this study. Hence, more functional SNPs of EGF and the mechanism of how rs2237051 SNP affecting GAgP should be investigated in the future.

\section{Conclusion}

In conclusion, we demonstrated that $E G F$ rs2237051 variant and the increased level of serum EGF were associated with the risk of GAgP, the serum EGF was up-regulated in patients with 
$264 \mathrm{GAgP}$. It was indicated that serum EGF might be a biomarker of GAgP and EGF rs2237051 may

265 be related to the genetic background of GAgP.

266

\section{Acknowledgments}

268 The study was funded by the National Natural Science Foundation of China, Beijing, China 269 (81271149). All authors have no conflict of interest statement.

270

271

272

273

274

275

276

277

278

279

280

281

282

283

284

285

286

287

288

289

290

\section{References}

Armitage GC. 1999. Development of a classification system for periodontal diseases and conditions. Annals of Periodontology 4:1-6.

Birkedal-Hansen H. 1993. Role of matrix metalloproteinases in human periodontal diseases. Journal of Periodontology 64:474-484.

Cardoso EM, Reis C, Manzanares-Céspedes MC.2018. Chronic periodontitis, inflammatory cytokines, and interrelationship with other chronic diseases. Postgraduate medicine 130(1):98-104.

Caroleo M., Carbone E. A., Greco,M., Corigliano D. M., Arcidiacono,B., Fazia G., Brunetti A. 2019. Brain-Behavior-Immune Interaction: Serum Cytokines and Growth Factors in Patients with Eating Disorders at Extremes of the Body Mass Index (BMI) Spectrum. Nutrients 11(9).

Chang KM, Lehrhaupt N, Lin LM, Feng J, Wu-Wang CY, Wang SL.1996. Epidermal growth factor in gingival crevicular fluid and its binding capacity in inflamed and noninflamed human gingiva. Archives of Oral Biology 41(7):719-24.

Cury PR, de Araujo VC, Canavez F, Furuse C, Leite KR, de Araujo NS. 2007. The effect of epidermal growth factor on matrix metalloproteinases and tissue inhibitors of metalloproteinase gene expression in cultured human gingival fibroblasts. Archives of Oral Biology 52:585-590.

Ekuni D, Yamamoto T, Koyama R, Tsuneishi M, Naito K, Tobe K. 2008. Relationship 
291 between body mass index and periodontitis in young Japanese adults. Journal of 292 Periodontal Research 43 (4): 417-421.

293 Hormia M, Thesleff I, Perheentupa J, Pesonen K, Saxen L. 1993. Increased rate of

294 salivary epidermal growth factor secretion in patients with juvenile periodontitis.

295 Scandinavian Journal of Dental Research 101:138-144.

296 Hosgood HD 3rd, Menashe I, Shen M, Yeager M, Yuenger J, Rajaraman P, He X,

297 Chatterjee N, Caporaso NE, Zhu Y, Chanock SJ, Zheng T, Lan Q. 2008. Pathway-based

298 evaluation of 380 candidate genes and lung cancer susceptibility suggests the

299 importance of the cell cycle pathway. Carcinogenesis 29:1938-1943.

300 Lyons JG, Birkedal-Hansen B, Pierson MC, Whitelock JM, Birkedal-Hansen H. 1993.

301 Interleukin-1 beta and transforming growth factor-alpha/epidermal growth factor

302 induce expression of M(r) 95,000 type IV collagenase/gelatinase and interstitial

303 fibroblast-type collagenase by rat mucosal keratinocytes. The Journal of biological

304 chemistry 268:19143-19151.

305 Mazza JE, Newman MG, Sims TN. 1981. Clinical and antimicrobial effect of stannous

306 fluoride on periodontitis. Journal of clinical periodontology 8:203-212.

307 Moosavijazi M, Rasouli Ghahroudi AA, Yaghoobee S, Bayani M, Salehi E,

308 Sadrimanesh R. 2014. Comparison of salivary epidermal growth factor levels in

309 patients with gingivitis and advanced periodontitis and healthy subjects. Journal of

310 Dentistry (Tehran, Iran) 11:516-522.

311 Moura-Grec PG, Marsicano JA, Carvalho CA, Carvalho Sales-Peres SH. 2014. Obesity

312 and periodontitis: systematic review and meta-analysis. Saude Colet 19 (6):1763-1772.

313 Oxford GE, Jonsson R, Olofsson J, Zelles T, Humphreys-Beher MG. 1999. Elevated

314 levels of human salivary epidermal growth factor after oral and juxtaoral surgery.

315 Journal of oral and maxillofacial surgery 57:154-158; discussion 158-159.

316 Ozer Yucel O, Berker E, Mesci L, Eratalay K, Tepe E, Tezcan I. 2015. Analysis of

317 TNF-alpha (-308) polymorphism and gingival crevicular fluid TNF-alpha levels in 
318 aggressive and chronic periodontitis: A preliminary report. Cytokine 72:173-177.

319 Pietruska MD, Pietruski JK, Stokowska W. 2000. Polypeptide growth factors in the

320 course of surgical periodontal treatment. Roczniki Akademii Medycznej Bialymstoku

321 45:199-210.

322 Quigley GA, Hein JW. 1962. Comparative cleansing efficiency of manual and power

323 brushing. Journal of the American Dental Association 65:26-29.

324 Suzuki A, Ji G, Numabe Y, Muramatsu M, Gomi K, Kanazashi M, Ogata Y, Shimizu

325 E, Shibukawa Y, Ito A, Ito T, Sugaya A, Arai T, Yamada S, Deguchi S, Kamoi K.

326 2004. Single nucleotide polymorphisms associated with aggressive periodontitis and

327 severe chronic periodontitis in Japanese. Biochemical and Biophysical Research

328 Communicatoins 317:887-892.

329 Vieira AR, Albandar JM. 2014. Role of genetic factors in the pathogenesis of

330 aggressive periodontitis. Periodontology 2000 65:92-106.

331 Yang PW, Hsieh MS, Huang YC, Hsieh CY, Chiang TH, Lee JM. 2014. Genetic

332 variants of EGF and VEGF predict prognosis of patients with advanced esophageal

333 squamous cell carcinoma. PLoS One 9:e100326.

334 Yucel-Lindberg T, Brunius G. 2006. Epidermal growth factor synergistically enhances

335 interleukin-8 production in human gingival fibroblasts stimulated with interleukin-1beta.

336 Archives of Oral Biology 51:892-898.

337 Zhan Z, Chen Y, Wu J, Zhang J, Tong S, Zhang C, Yang Y. 2013. Functional epidermal

338 growth factor gene polymorphisms and risk of gastric cancer. Oncology Letters 5:631339636. 


\section{Table 1 (on next page)}

Characteristics, genotypes of EGF rs2237051 and serum EGF levels in GAgP patients and healthy controls. 
1 Table 1. Characteristics, genotypes of $E G F$ rs2237051 and serum EGF levels in GAgP patients and healthy 2 controls.

\begin{tabular}{|c|c|c|c|}
\hline Risk of GAgP & GAgP Patients & Healthy Controls & P-value \\
\hline $\mathrm{N}$ & 216 & 138 & \\
\hline Age, $y$ & $27.29 \pm 4.25$ & $27.10 \pm 4.23$ & 0.688 \\
\hline Gender & & & 0.859 \\
\hline female & $85(39.35 \%)$ & $53(38.41 \%)$ & \\
\hline male & $131(60.65 \%)$ & $85(61.59 \%)$ & \\
\hline BMI, $\mathrm{Kg} / \mathrm{m}^{2}$ & $22.10 \pm 3.52$ & $21.28 \pm 3.06$ & $0.026^{*}$ \\
\hline BMI categories & & & $0.002^{*}$ \\
\hline$<18.5$ & $26(12.32 \%)$ & $20(14.60 \%)$ & \\
\hline$>=18.5,<24.9$ & $138(65.40 \%)$ & $106(77.37 \%)$ & \\
\hline$>=24.9$ & $47(22.27 \%)$ & $11(8.03 \%)$ & \\
\hline mean PLI & $2.34 \pm 0.39$ & $1.40 \pm 0.46$ & $<0.001^{*}$ \\
\hline mean PD, mm & $4.82 \pm 0.98$ & $1.89 \pm 0.61$ & $<0.001^{*}$ \\
\hline mean BI & $3.55 \pm 0.47$ & $1.17 \pm 0.37$ & $<0.001^{*}$ \\
\hline mean $\mathrm{AL}, \mathrm{mm}$ & $4.29 \pm 1.44$ & $0.01 \pm 0.52$ & $<0.001^{*}$ \\
\hline serum EGF, pg/ml & $46.77 \pm 9.38$ & $33.54 \pm 8.62$ & $<0.001 *$ \\
\hline Alleles of rs 2237051 & & & $0.005^{*}$ \\
\hline A & $311(71.99 \%)$ & $171(61.96 \%)$ & \\
\hline G & $121(28.01 \%)$ & $105(38.04 \%)$ & \\
\hline Genotype of rs2237051 & & & $0.009^{*}$ \\
\hline GG & $12(5.56 \%)$ & $19(13.77 \%)$ & \\
\hline $\mathrm{AG}$ & $97(44.91 \%)$ & $67(48.55 \%)$ & \\
\hline AA & $107(49.54 \%)$ & $52(37.68 \%)$ & \\
\hline Genotype of rs 2237051 & & & $0.029^{*}$ \\
\hline $\mathrm{AG} / \mathrm{GG}$ & $109(50.46 \%)$ & $86(62.32 \%)$ & \\
\hline AA & $107(49.54 \%)$ & $52(37.68 \%)$ & \\
\hline Genotype of rs2237051 & & & $0.008^{*}$ \\
\hline GG & $12(5.56 \%)$ & $19(13.77 \%)$ & \\
\hline $\mathrm{AG} / \mathrm{AA}$ & $204(94.44 \%)$ & $119(86.23 \%)$ & \\
\hline
\end{tabular}

3 GAgP, generalized aggressive periodontitis; BMI: body mass index; PLI: plaque index; PD: probing depth; BI,

4 bleeding index; AL, attachment loss; EGF, epidermal growth factor.

5 Results in table: Mean+SD / N (\%);

$6 \quad{ }^{*} \mathrm{P}<0.05$, statistical significance 
Table 2 (on next page)

Univariate analysis for risk of GAgP. 
1 Table 2. Univariate analysis for risk of GAgP.

\begin{tabular}{|c|c|c|c|}
\hline Variables & Statistics & $\begin{array}{l}\text { Risk of GAgP } \\
\text { OR }(95 \% \mathrm{CI})\end{array}$ & P-value \\
\hline Age, y & $27.21 \pm 4.24$ & $1.01(0.96,1.06)$ & 0.6875 \\
\hline \multicolumn{4}{|l|}{ Gender } \\
\hline female & $138(38.98 \%)$ & Ref. & \\
\hline male & $216(61.02 \%)$ & $0.96(0.62,1.49)$ & 0.8587 \\
\hline BMI, $\mathrm{Kg} / \mathrm{m}^{2}$ & $21.78 \pm 3.37$ & $1.08(1.01,1.15)$ & $0.0279 *$ \\
\hline \multicolumn{4}{|l|}{ BMI categories } \\
\hline$<18.5$ & $46(13.22 \%)$ & Ref. & \\
\hline$>=18.5,<24.9$ & $244(70.11 \%)$ & $1.00(0.53,1.89)$ & 0.9964 \\
\hline$>=24.9$ & $58(16.67 \%)$ & $3.29(1.37,7.91)$ & $0.0079^{*}$ \\
\hline Alleles of rs 2237051 & & & $0.0054 *$ \\
\hline $\mathrm{A}$ & $482(68.08 \%)$ & Ref. & \\
\hline G & $226(31.92 \%)$ & $0.63(0.46,0.87)$ & \\
\hline \multicolumn{4}{|l|}{ Genotype of rs2237051 } \\
\hline GG & $31(8.76 \%)$ & Ref. & \\
\hline $\mathrm{AG}$ & $164(46.33 \%)$ & $2.29(1.04,5.04)$ & $0.0388^{*}$ \\
\hline AA & $159(44.92 \%)$ & $3.26(1.47,7.22)$ & $0.0036^{*}$ \\
\hline \multicolumn{4}{|c|}{ Genotype of rs2237051 } \\
\hline $\mathrm{AG} / \mathrm{GG}$ & $195(55.08 \%)$ & Ref. & \\
\hline AA & $159(44.92 \%)$ & $1.62(1.05,2.51)$ & $0.0292^{*}$ \\
\hline serum EGF, pg/ml & $41.61 \pm 11.14$ & $1.17(1.13,1.21)$ & $<0.0001^{*}$ \\
\hline
\end{tabular}

2 Data were presented as OR $(95 \% \mathrm{CI})$, outcome: Risk of GAgP.

3 Exposure: Age; BMI; BMI categories; Gender; genotype of rs2237051; serum EGF.

$4 \quad{ }^{*} \mathrm{P}<0.05$, statistical significance. 


\section{Table 3 (on next page)}

Multiple regressions of EGF rs2237051 and serum EGF for risk of GAgP. 
1 Table 3. Multiple regressions of EGF rs2237051 and serum EGF for risk of GAgP.

\begin{tabular}{lclcl}
\hline Exposure & Adjust I & P-value & Adjust II & P-value \\
& OR $(95 \% \mathrm{CI})$ & & OR $(95 \% \mathrm{CI})$ & \\
\hline
\end{tabular}

Genotype of

rs2237051

AG/GG (N=195) Ref.

Ref.

AA $(\mathrm{N}=159) \quad 1.64(1.06,2.54) \quad 0.0263^{*} \quad 1.65(1.06,2.57) \quad 0.0264^{*}$

$\begin{array}{llllll}\text { serum EGF, pg/ml } & 1.17(1.13,1.21) & <0.0001^{*} & 1.18(1.14,1.22) & <0.0001^{*}\end{array}$

2 Data were presented as OR $(95 \% \mathrm{CI})$, outcome: Risk of GAgP.

3 Exposure: rs2237051; serum EGF.

4 Model I was adjusted for age and gender.

5 Model II was adjusted for age, gender and BMI.

$6 \quad{ }^{*} \mathrm{P}<0.05$, statistical significance. 


\section{Table 4 (on next page)}

The association between genotype of rs2237051 and serum EGF. 
1 Table 4. The association between genotype of rs2237051 and serum EGF.

\begin{tabular}{llll}
\hline \multirow{2}{*}{ Exposure } & Non-adjusted & Model I & Model II \\
& $\beta(95 \% \mathrm{CI})$ & $\beta(95 \% \mathrm{CI})$ & $\beta(95 \% \mathrm{CI})$ \\
\hline
\end{tabular}

\section{Total}

Genotype of rs2237051

$\begin{array}{llll}\text { AG/GG } & \text { Ref. } & \text { Ref. } & \text { Ref. } \\ \text { AA } & 3.06(1.06,5.06)^{*} & 2.98(0.98,4.98)^{*} & 2.86(0.88,4.84)^{*}\end{array}$

\section{Healthy Controls}

Genotype of rs2237051

$\begin{array}{llll}\text { AG/GG } & \text { Ref. } & \text { Ref. } & \text { Ref. } \\ \text { AA } & -0.33(-3.41,2.76) & -0.22(-3.32,2.89) & 0.11(-2.97,3.19)\end{array}$

\section{GAgP Patients}

Genotype of rs 2237051

$\begin{array}{llll}\mathrm{AG} / \mathrm{GG} & \text { Ref. } & \text { Ref. } & \text { Ref. } \\ \mathrm{AA} & 5.10(2.52,7.68) * & 4.97(2.35,7.59) * & \mathbf{4 . 7 0}(2 .\end{array}$

AA $\quad 5.10(2.52,7.68) * \quad 4.97(2.35,7.59) * \quad \mathbf{4 . 7 0}(2.09,7.31) *$

2 Data were presented as $\beta(95 \% \mathrm{CI})$, outcome: serum EGF, exposure: genotype of rs 2237051 .

3 Model I was adjusted for age and gender.

4 Model II was adjusted for age gender and BMI.

$5 * \mathrm{P}<0.05$, statistical significance. 